\title{
Características da reprodução de tartarugas marinhas (Testudines, Cheloniidae) no litoral sul da Bahia, Brasil
}

\author{
Cássia Santos Camillo ${ }^{1,2}$, Renato de Mei Romero ${ }^{1}$, Luciano Gerolim Leone ${ }^{1}$, \\ Renata Lucia Guedes Batista ${ }^{1}$, Raquel Sá Velozo ${ }^{1}$ \& Sérgio Luiz Gama Nogueira-Filho ${ }^{1}$ \\ ${ }^{1}$ Programa de Pós-graduação em Zoologia, Universidade Estadual de Santa Cruz - UESC, \\ Rod. Ilhéus-Itabuna Km 16, CEP 45662-000, Ilhéus, Bahia, Brasil, http://www.uesc.br/zoologia \\ ${ }^{2}$ Autor para correspondência: Cássia Camillo, cassiacamillo@gmail.com
}

CAMILLO, C.S., ROMERO, R. M., LEONE, L. G., BATISTA, R.L.G., VELOZO, R.S. \& NOGUEIRA-FILHO, S.L.G. Reproduction of sea turtles (Testudines, Cheloniidae) in the Southern Coast of Bahia, Brazil. Biota Neotrop., 9(2): http://www.biotaneotropica.org.br/v9n2/en/abstract?article+bn02209022009.

\begin{abstract}
Few studies have been carried out on nesting of sea turtles in the southern coast of Bahia, an area that is experiencing a fast process of tourist occupation what can increase levels of threat to these species. Thus, this study aimed to identify sea turtle species that nest in the area and to describe the main characteristics of their clutches. During four reproductive seasons, from 2004 to 2008, we identified and monitored nests found at beaches located between the municipalities of Uruçuca and Itacaré, in the southern coast of Bahia. The collected variables were: clutch size, incubation duration, hatching success and stages of embryonic mortality. We registered an average of $37 \pm 6.2$ nests per season, with nests of Caretta caretta and Eretmochelys imbricata being more frequent, along with sporadic nests of Chelonia mydas. Nesting season of $C$. caretta extends from October to January and that of E. imbricata from December to March. This temporary displacement of reproductive seasons might be related to a selective adjustment to minimize competition among species. Besides the time of nesting, the two species differed also in clutch size, but they did not differ in incubation duration and in hatching success. According to our results, we believe that areas with low concentration of nests should also be a target of conservation programs, due to their relevance to the increase of genotypic, and specially fenotypic, variation of sea turtle species, so that Brazilian populations can adapt and survive to the constant threats. Those conservation programs can be established by partnerships between non-governmental organizations and private institutions and the Brazilian government institution for the conservation of sea turtles (Centro TAMAR/ICMBio).

Keywords: loggerhead sea turtle, hawksbill sea turtle, hatching success, incubation duration, APA Itacaré-Serra Grande.
\end{abstract}

CAMILLO, C.S., ROMERO, R.M., LEONE, L.G., BATISTA, R.L.G., VELOZO, R.S. \& NOGUEIRA-FILHO, S.L.G. Características da reprodução de tartarugas marinhas (Testudines, Cheloniidae) no litoral sul da Bahia, Brasil. Biota Neotrop., 9(2): http://www.biotaneotropica.org.br/v9n2/pt/abstract?article+bn02209022009.

Resumo: Poucos estudos foram realizados sobre a nidificação de tartarugas marinhas no litoral sul da Bahia, região que está passando por um processo rápido de ocupação turística que pode ameaçar ainda mais estas espécies. Por estes motivos, este estudo teve como objetivos identificar as espécies de tartarugas marinhas que desovam no local e descrever as principais características de suas ninhadas. Durante quatro temporadas reprodutivas, de 2004 a 2008, foram identificados e monitorados os ninhos encontrados nas praias localizadas entre Uruçuca e Itacaré no litoral sul da Bahia. As variáveis coletadas foram: tamanho de ninhada, duração da incubação, sucesso de eclosão e fases de mortalidade embrionária. Foi registrada uma média de $37 \pm 6,2$ ninhos por temporada, com maior freqüência de ninhos das espécies Caretta caretta e Eretmochelys imbricata, tendo sido registradas também desovas esporádicas de Chelonia mydas. A temporada de desova de $C$. caretta ocorre de outubro a janeiro e de E. imbricata de dezembro a março. Este deslocamento temporal das temporadas reprodutivas pode estar relacionado a uma adaptação para minimizar a competição interespecífica. Além da época de nidificação, as duas espécies diferiram no tamanho de suas ninhadas, mas não diferiram na duração da incubação e no sucesso de eclosão. Diante dos resultados, acredita-se que áreas com baixa concentração de ninhos também devem ser alvo de programas de conservação, devido à sua relevância para a ampliação da variação genotípica e, sobretudo, fenotípica, das espécies de tartarugas marinhas, de forma que as mesmas possam se adaptar e sobreviver às constantes ameaças às quais estão submetidas. Tais programas de conservação podem ser estabelecidos por meio de parcerias entre organizações não governamentais e instituições privadas e a instituição governamental responsável pela conservação das tartarugas marinhas (Centro TAMAR/ICMBio).

Palavras-chave: tartaruga cabeçuda, tartaruga de pente, sucesso de eclosão, duração da incubação, APA ItacaréSerra Grande. 


\section{Introdução}

O litoral sul da Bahia encontra-se inserido nas áreas de nidificação dos quelônios Caretta caretta (Linnaeus, 1758) e Eretmochelys imbricata (Linnaeus, 1766) no Brasil, sendo a primeira a espécie de tartaruga marinha que apresenta maior número de ninhos no litoral brasileiro (Marcovaldi \& Marcovaldi 1999). As áreas freqüentes de nidificação desta espécie estendem-se do litoral do Rio de Janeiro ao litoral do Sergipe, sendo o litoral norte da Bahia a área de maior concentração de seus ninhos (Marcovaldi \& Laurent 1996, Marcovaldi \& Marcovaldi, 1999). Por sua vez, apesar de registros de nidificação de E. imbricata estenderem-se do litoral do Espírito Santo ao Rio Grande do Norte, esta espécie desova, regularmente, sobretudo no litoral norte da Bahia (Marcovaldi et al. 1999, 2007).

Além dessas, as espécies Chelonia mydas (Linnaeus, 1758), Lepidochelys olivacea (Eschscholtz, 1829) e Dermochelys coriacea (Linnaeus, 1766), também utilizam praias brasileiras para nidificação (Marcovaldi \& Marcovaldi 1999). Enquanto C. mydas possui grandes colônias reprodutivas nas ilhas oceânicas de Atol das Rocas e Trindade e, em menor densidade, no arquipélogo de Fernando de Noronha, L. olivacea desova no litoral norte da Bahia e Sergipe e D. coriacea no litoral do Espírito Santo (Marcovaldi \& Marcovaldi 1999).

As cinco espécies de tartarugas marinhas que ocorrem no Brasil são mencionadas na Lista Nacional das Espécies da Fauna Brasileira Ameaçadas de Extinção: $C$. mydas e $C$. caretta estão listadas na categoria "vulnerável", L. olivacea e E. imbricata como "em perigo" e D. coriacea como "criticamente em perigo" (IBAMA 2003).

As características das ninhadas de tartarugas marinhas, como número de ovos, duração da incubação e sucesso de eclosão podem variar de acordo com características das fêmeas e com características das praias de nidificação (Miller 1997). Por estes motivos, o conhecimento de tais aspectos da nidificação pode auxiliar no estabelecimento de planos de manejo e conservação dessas espécies nas diferentes áreas de desova. Estudos prévios sobre a nidificação de tartarugas marinhas no Brasil foram realizados no litoral do estado do Rio Grande do Norte (Marcovaldi et al. 2007), no litoral do estado da Paraíba (Mascarenhas et al. 2003), litoral norte do estado da Bahia (Marcovaldi \& Laurent 1996, Marcovaldi et al. 1997, Godfrey et al. 1999, Marcovaldi et al. 1999) e no litoral do Espírito Santo (Baptistotte et al. 1999, 2003, Ferreira-Junior et al. 2003, Thomé et al. 2007). Pouco se sabe, portanto, sobre as colônias reprodutivas em outras áreas. Neste contexto, os objetivos deste estudo foram identificar as espécies de tartarugas marinhas que nidificam em um trecho do litoral sul da Bahia e descrever as características principais de suas ninhadas.

\section{Material e Métodos}

\section{1. Área de estudo}

Este estudo foi realizado nas praias Pompilho, Patizeiro e Itacarezinho, localizadas no litoral sul do estado da Bahia, Brasil, pertencentes ao município de Uruçuca ( $14^{\circ} 35^{\prime} \mathrm{S}$ e $\left.39^{\circ} 16^{\prime} \mathrm{O}\right)$ e Ita-

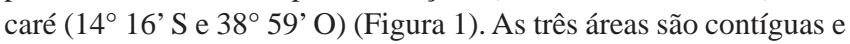
totalizam aproximadamente $11 \mathrm{~km}$ de extensão, sendo limitadas por a oramentos rochosos tanto ao norte quanto ao sul. As praias são diretamente in uenciadas pelas condições do oceano, dominadas por ondas e sem grande in uência de estuários e rios, apesar da existência da foz do rio Tijuípe entre as praias do Pompilho e do Patizeiro (T.K. Rodrigues, dados não publicados). Suas características morfodinâmicas apresentam uma mudança gradativa no continuum re etivo-dissipativo de sul para norte. Quanto à ocupação humana, existem poucas construções permanentes, porém o uxo de pescadores artesanais nas praias é freqüente.

\section{Coleta de dados}

Na costa brasileira, a reprodução de tartarugas marinhas estendese de setembro a março. Durante as temporadas de nidificação dos anos 2004/2005, 2006/2007 e 2007/2008, as três praias foram monitoradas diariamente. Durante a temporada de 2005/2006, devido a problemas logísticos, os monitoramentos foram realizados de janeiro a março e apenas nas praias do Patizeiro e Itacarezinho.

A área de estudo foi percorrida a pé, com o objetivo de localizar, identificar e marcar ninhos de tartarugas marinhas. Os ninhos eram localizados por meio dos rastros na areia deixados pelas fêmeas. Após a localização da câmara de ovos, os ninhos foram marcados com estacas padrão e acompanhados diariamente para verificação e registro de possíveis perturbações, como predação por humanos, animais silvestres ou domésticos, erosão pela maré e perda de estaca. Durante as temporadas de 2004/2005 e 2005/2006, ninhos localizados em áreas de risco de erosão pela maré foram transferidos para áreas consideradas seguras na mesma praia $(\mathrm{N}=5)$. Apenas ninhos, cuja espécie foi identificada, que foram mantidos in situ e que chegaram ao final do período de incubação sem que tenha ocorrido alguma destas perturbações foram incluídos nas análises $(84,8 \%)$.

Ao serem visualizados os rastros dos filhotes na praia ou após 70 dias de incubação, os ninhos foram abertos para identificação das espécies e coleta de variáveis biológicas. Foram analisadas as seguintes variáveis: a) tamanho da ninhada: corresponde ao número total de ovos viáveis no ninho; foram excluídos deste total os ovos que apresentavam anomalias (sem vitelo ou com formato e tamanho anormais); b) duração da incubação: considerado desde o dia da desova até o dia da emergência dos filhotes, quando seus rastros foram visualizados na praia; c) sucesso de eclosão ou filhotes vivos: corresponde ao número total de filhotes vivos, estimados a partir da contagem das cascas, dividido pelo tamanho da ninhada, foram incluídos neste total os filhotes vivos retidos no ninho, os quais foram liberados na praia; d) filhotes natimortos: número de filhotes mortos dentro do ninho após a eclosão dividido pelo tamanho da ninhada; foram incluídos também filhotes mortos que perfuraram a casca do ovo, mas não a deixaram; e) ovos não eclodidos.

Durante as temporadas de 2004/2005, 2006/2007 e 2007/2008, os ovos não eclodidos foram ainda subdivididos em quatro categorias de mortalidade embrionária, de acordo com a classificação de Eckert \& Eckert (1990): a) ovos não desenvolvidos: aqueles que não apresentavam sinais de desenvolvimento embrionário; b) embriões mortos em fase embrionária inicial (fase 1): aqueles com embriões menores que $10 \mathrm{~mm}$ ou sinais de formação de sangue; c) embriões mortos em fase embrionária intermediária (fase 2): ovos contendo embriões de tamanhos entre 10 e $30 \mathrm{~mm}$; d) embriões mortos em fase embrionária tardia (fase 3): ovos com embriões com mais de $30 \mathrm{~mm}$ de comprimento.

\section{Análise dos dados}

Para o cálculo da duração da incubação foram utilizados apenas os dados dos ninhos em que foram visualizados os rastros dos filhotes - indicativos de sua emersão. As médias das variáveis biológicas registradas para as diferentes espécies foram comparadas por meio do teste de Mann-Whitney. As médias das categorias de mortalidade embrionária - número de filhotes natimortos, de ovos não desenvolvidos, de embriões fases 1, 2 e 3 - foram comparadas pelo teste de Kruskal-Wallis, seguido pelo teste a posteriori do método de Dunn, para verificar em quais delas a mortalidade foi maior. Para todas as análises utilizou-se o programa BioEstat (versão 4.0), sendo empregado o nível de significância 0,05 . 


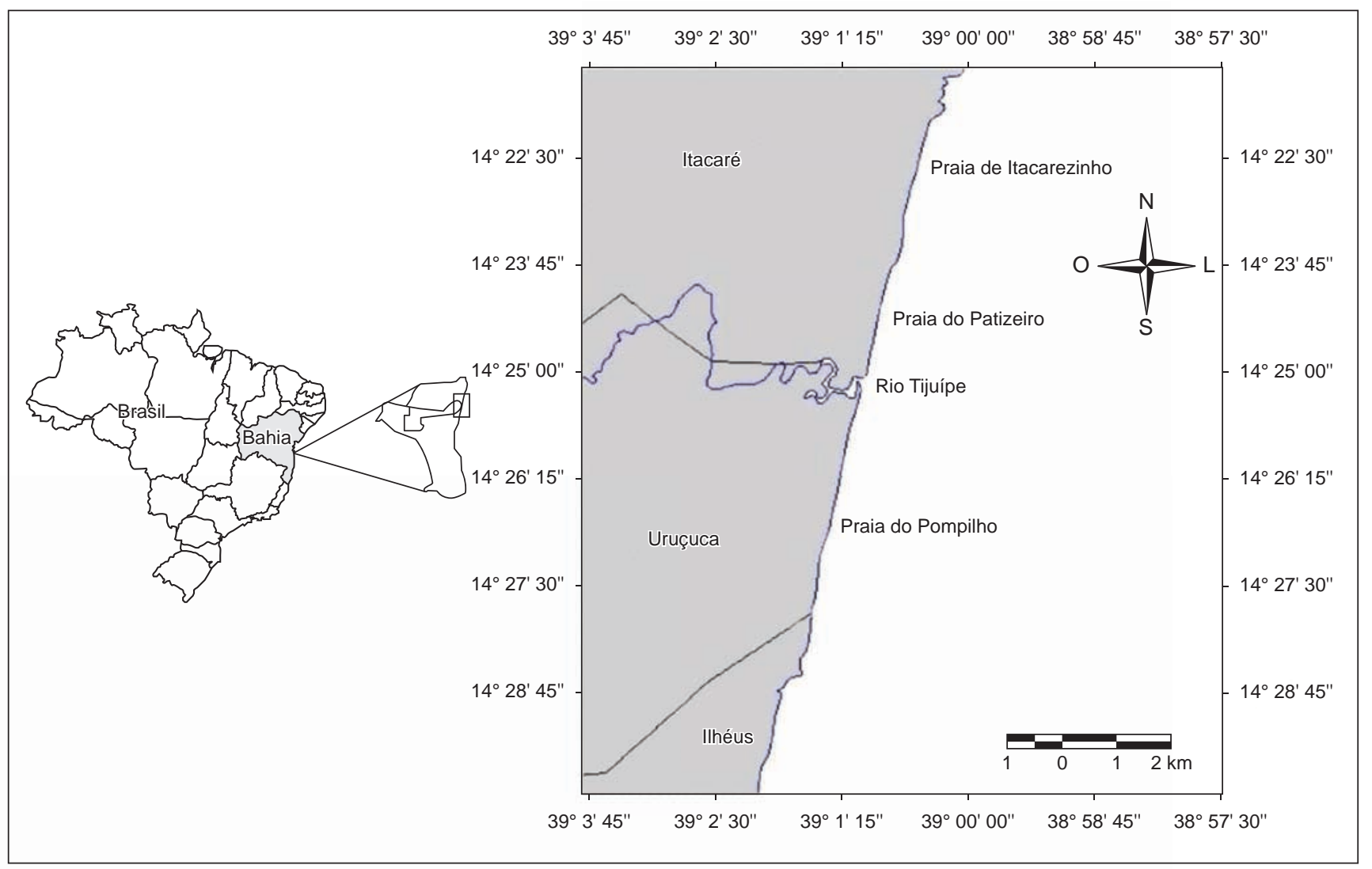

Figura 1. Mapa de localização das praias de Pompilho, Patizeiro e Itacarezinho - área de estudo - nos municípios de Itacaré e Uruçuca, Bahia, Brasil.

Figure 1. Map of the localization of Pompilho, Patizeiro and Itacarezinho beaches in the municipalities of Itacaré and Uruçuca, Bahia State, Brazil.

\section{Resultados}

Considerando-se apenas as temporadas reprodutivas de 2004/2005, 2006/2007 e 2007/2008, nas quais o monitoramento foi realizado em toda a área de estudo e diariamente, foi registrada uma média de $37 \pm 6,2$ ninhos por temporada, o que representa uma densidade menor do que quatro ninhos por quilômetro de praia. Do total de ninhos registrados nestas três temporadas $(\mathrm{N}=111), 85,6 \%$ foram identificados; destes, $52,6 \%$ pertenciam à espécie $C$. caretta e $44,2 \%$ à E. imbricata. Além dessas duas espécies, foram identificados ninhos de C. mydas: um na temporada de 2004/2005 e dois na de 2007/2008. Para alguns ninhos $(14,4 \%)$ não foi possível identificar a espécie devido à predação, à erosão ocasionada pela maré ou à ausência de filhotes natimortos ou embriões mortos (>30 mm), no momento da abertura, que possibilitassem a identificação da espécie.

Neste trecho do litoral sul da Bahia, em todos os anos estudados, as temporadas de nidificação iniciaram-se em outubro, com os primeiros ninhos de $C$. caretta, e se estenderam até abril, com os ninhos de E. imbricata. As temporadas de reprodução das duas espécies diferiram: enquanto para $C$. caretta os meses de maior concentração de ninhos foram os de outubro a janeiro, para E. imbricata foram os de dezembro a março (Figura 2).

Os tamanhos médios das ninhadas também diferiram entre estas duas espécies $(\mathrm{U}=437 ; \mathrm{P}<0,01)$. Para $C$. caretta a média e desvio padrão foram de $94,4 \pm 27,6$ ovos $(\mathrm{N}=45,20-146)$ e para E. imbricata foram de 118,3 $\pm 23,6$ ovos ( $\mathrm{N}=38,70-196$; Figura 3 a). A duração média da incubação, por outro lado não diferiu entre as duas espécies $(\mathrm{U}=297 ; \mathrm{P}=0,08$; Figura $3 \mathrm{~b})$ : 56,9 \pm 3,6 dias para C. caretta $(\mathrm{N}=31,50-65$ dias $)$ e 58,3 $\pm 3,3$ dias para $E$. imbricata ( $\mathrm{N}=26,50-63$ dias $)$.

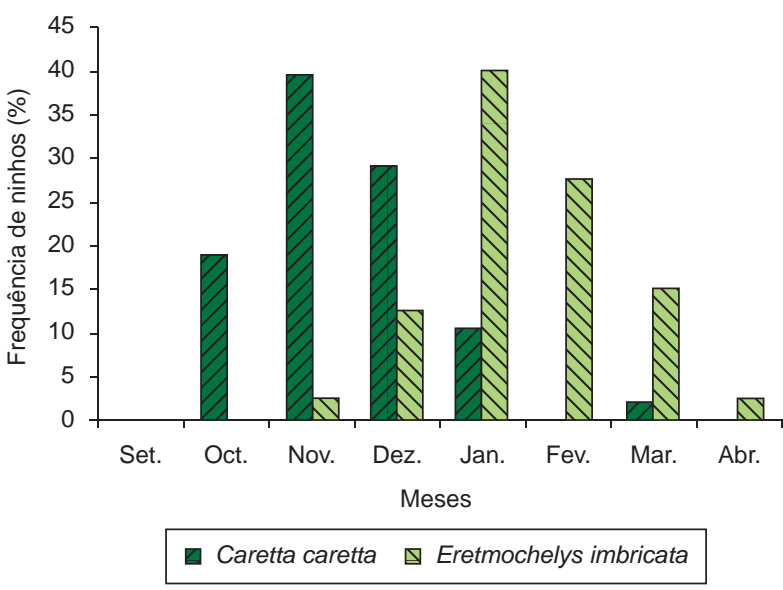

Figura 2. Distribuição dos ninhos de C. caretta $(\mathrm{N}=48)$ e E. imbricata ( $\mathrm{N}=40)$ ao longo das temporadas de nidificação nas praias de Pompilho, Patizeiro e Itacarezinho, no litoral sul da Bahia, considerando-se as temporadas 04/05, 06/07 e 07/08.

Figure 2. Distribution of $C$. caretta and E. imbricata nests during the nesting season in Pompilho, Patizeiro and Itacarezinho beaches, considering the 04/05, 06/07 and 07/08 nesting seasons.

As tartarugas $C$. caretta e E. imbricata também não diferiram quanto ao sucesso de eclosão $(\mathrm{U}=693 ; \mathrm{P}=0,14)$. O sucesso médio de eclosão nos ninhos de $C$. caretta foi de $68,6 \pm 27,5 \%$ ( $\mathrm{N}=45,0-97,1)$, enquanto que nos ninhos de E. imbricata foi de $78,3 \pm 18,7 \%(\mathrm{~N}=38,14,3-98,0 ;$ Figura $4 a)$. Por outro lado, as duas 
Tabela 1. Médias e desvios padrão (DP) das categorias de mortalidade embrionária para os ninhos de C. caretta e E. imbricata nas praias de Pompilho, Patizeiro e Itacarezinho no litoral sul da Bahia, durante as temporadas reprodutivas de 04/05, 06/07 e 07/08. Letras diferentes indicam médias diferentes, de acordo com o teste de Kruskal-Wallis, seguido pelo teste a posteriori de Dunn $(\mathrm{P}<0,05)$.

Table1. Means and standard deviations of the embryonic mortality categories for $C$. caretta and E. imbricata nests in Pompilho, Patizeiro and Itacarezinho beaches, southern coast of Bahia, during 04/05, 06/07 and 07/08 nesting seasons. Different letters indicate means statistically different, according to Kruskal-Wallis test, followed by tDunn a posteriori method.

\begin{tabular}{lcccc}
\hline $\begin{array}{c}\text { Categoria de mortalidade } \\
\text { embrionária }\end{array}$ & \multicolumn{2}{c}{$\begin{array}{c}\text { Caretta } \\
\text { caretta }\end{array}$} & \multicolumn{2}{c}{$\begin{array}{c}\text { Eretmochelys } \\
\text { imbricata }\end{array}$} \\
\hline & Média & DP & Média & DP \\
\hline Ovos não desenvolvidos & $15,8^{\mathrm{a}}$ & 19,1 & $7,5^{\mathrm{a}}$ & 7,2 \\
Embriões em fase 1 & $4,3^{\mathrm{bc}}$ & 6,1 & $4,3^{\mathrm{b}}$ & 7,0 \\
Embriões em fase 2 & $1,6^{\mathrm{b}}$ & 3,0 & $0,6^{\mathrm{c}}$ & 1,1 \\
Embriões em fase 3 & $7,1^{\mathrm{c}}$ & 17,1 & $3,1^{\mathrm{b}}$ & 2,8 \\
\hline
\end{tabular}
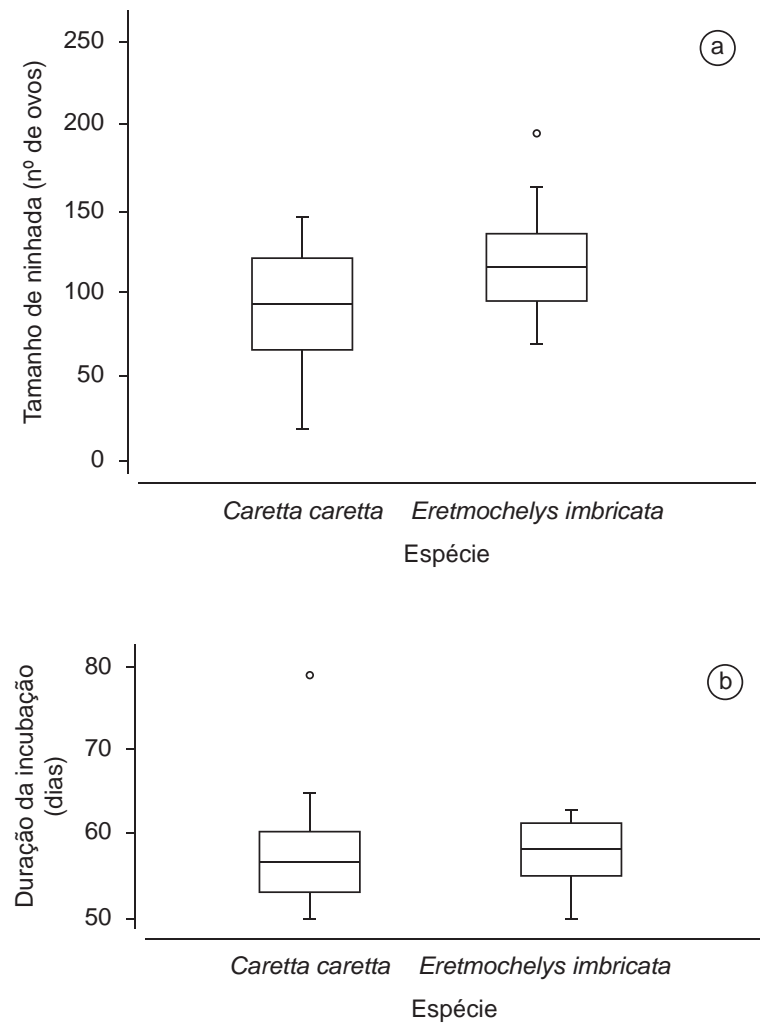

Figura 3. a) Tamanho médio de ninhada e b) duração média da incubação para os ninhos de C. caretta e E. imbricata nas praias de Pompilho, Patizeiro e Itacarezinho, no litoral sul da Bahia, considerando as temporadas de 2004/2005, 2005/2006, 2006/2007 e 2007/2008. As caixas representam as médias e os desvios padrão, as suíças (whiskers) representam os limites máximos e mínimos e os círculos, os valores extremos.

Figure 3. a) Mean clutch sizes and b) mean incubation duration for $C$. caretta and E. imbricata nests during the nesting season in Pompilho, Patizeiro and Itacarezinho beaches, southern coast of Bahia, considering the 04/05, 05/06, 06/07 and 07/08 nesting seasons. Boxes represent means and standard deviations, whiskers represent maximum and minimum values and circles represent extreme values.
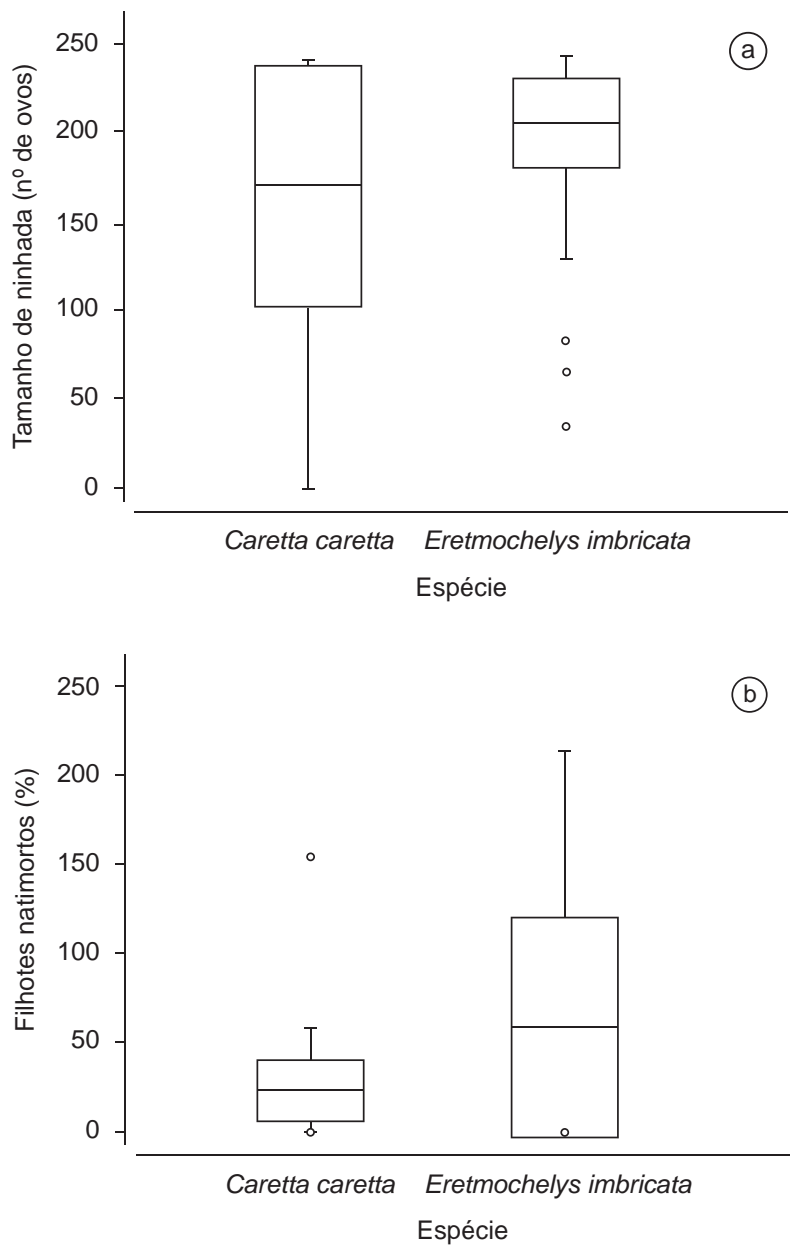

Figura 4. a) Sucesso de eclosão e b) proporção de filhotes natimortos para os ninhos de C. caretta e E. imbricata nas praias de Pompilho, Patizeiro e Itacarezinho no litoral sul da Bahia, considerando as temporadas de 04/05, 05/06, 06/07 e 07/08. As caixas representam as médias e os desvios padrão, as suíças (whiskers) representam os limites máximos e mínimos e os círculos, os valores extremos.

Figure 4. a) Hatching success and b) proportion of dead hatchlings for C. caretta and E. imbricata nests during the nesting season in Pompilho, Patizeiro and Itacarezinho beaches, southern coast of Bahia, considering the 04/05, 05/06, 06/07 and 07/08 nesting seasons. Boxes represent means and standard deviations, whiskers represent maximum and minimum values and circles represent extreme values.

espécies diferiram quanto à proporção de filhotes natimortos, a qual foi menor para C. caretta $(2,9 \pm 4,22 \%)$ do que para E. imbricata $(7,0 \pm 11,7 \% ; \mathrm{U}=582,5, \mathrm{P}=0,01$; Figura $4 \mathrm{~b})$.

Ao comparar as diferentes fases de mortalidade embrionária para cada espécie, verificou-se que para ambas as espécies houve uma maior proporção de ovos não desenvolvidos e uma menor proporção de ovos não eclodidos contendo embriões mortos em fase embrionária intermediária - fase 2 (Tabela 1). Comparando as duas espécies, estas não diferiram quanto à proporção de ovos não desenvolvidos ( $\mathrm{U}=565,0, \mathrm{P}=0,06$ ), ou na proporção de embriões mortos nas demais fases (fase 1: $\mathrm{U}=741,0, \mathrm{P}=0,94$; fase 2 : $\mathrm{U}=641,5, \mathrm{P}=0,28$; fase 3: $U=726,0, P=0,82$ ).

Como foram registrados apenas três ninhos de C. mydas os dados não foram utilizados nas análises anteriores. Na temporada dos anos 2004/2005 foi registrado apenas um ninho desta espécie, depositado 
Tabela 2. Comparação entre as variáveis dos ninhos de C. caretta, E. imbricata e $C$. mydas em diversos estudos no Brasil.

Table 2. Comparison among nest variables of C. caretta, E. imbricata and C. mydas in different studies carried out in Brazil.

\begin{tabular}{lcccc}
\hline Espécie & $\begin{array}{c}\text { Tamanho da ninhada } \\
\left(\mathbf{n}^{\mathbf{0}} \text {. de ovos) }\right.\end{array}$ & $\begin{array}{c}\text { Duração da } \\
\text { incubação (dias) }\end{array}$ & $\begin{array}{c}\text { Sucesso de eclosão } \\
(\%)\end{array}$ & Autor(es) \\
\hline Caretta caretta & 94,4 & 56,9 & 68,6 & Presente estudo \\
& 126,7 & 53,2 & 73,1 & Marcovaldi \& Laurent (1996) \\
Eretmochelys imbricata & - & 50,1 a 51,3 & 70,7 a 78,1 & TAMAR (2006)* \\
& 109,4 & 58,5 & 72,6 & Almeida \& Mendes (2007) \\
& 118,3 & 58,3 & 78,3 & Presente estudo \\
& 140,0 & 55,1 & 61,0 & Marcovaldi \& Laurent (1996) \\
Chelonia mydas & - & 51,2 a 54,1 & 47,1 a 56,1 & TAMAR (2006)* \\
& 133,9 & - & 80 & Mascarenhas et al. (2003) \\
& 136,4 & 55,9 a 62,9 & 51,5 a 54,7 & Marcovaldi et al. (1999)* \\
& 111,0 & 50,0 e 52,0 & 85,4 & Presente estudo \\
& 127,8 & - & 75,4 & Marcovaldi \& Laurent (1996) \\
\hline
\end{tabular}

Tabela 2. São apresentadas a maior e a menor média registradas para as bases do Projeto TAMAR/ICMBio do litoral norte da Bahia.

no dia 07/01/2005, com 112 ovos, que teve uma duração de incubação de 52 dias e o sucesso de eclosão de $88,4 \%$. Na temporada de $07 / 08$, foram registrados 2 ninhos, um depositado no dia 15/01/2008, com 125 ovos e outro, depositado no dia 26/01/2008, com 96 ovos. A duração da incubação foi de 50 dias para o primeiro, sendo que para o segundo não foi possível estimar, pois os rastros dos filhotes não foram visualizados. O sucesso de eclosão de foi de 76,0 e 91,7 \%.

\section{Discussão}

A época de nidificação das espécies $C$. caretta e E. imbricata no litoral sul da Bahia manteve-se similar à registrada no litoral norte da Bahia (Marcovaldi \& Laurent 1996, Marcovaldi et al. 1999), região com maior número de ninhos de tartarugas marinhas no Brasil, com uma média de 26,2 ninhos por quilômetro de praia (TAMAR, 2006). Estudos prévios relataram a mesma diferença encontrada para os picos de nidificação das temporadas reprodutivas de $C$. caretta e E. imbricata (Marcovaldi \& Laurent, 1996, Marcovaldi et al. 1999). Esta diferença pode estar relacionada a uma adaptação para minimizar a competição interespecífica, por meio de deslocamento temporal das temporadas reprodutivas.

Além da diferença para o início de temporada, as tartarugas marinhas das espécies $C$. caretta e E. imbricata diferiram também quanto ao tamanho de suas ninhadas. A mesma diferença entre estas espécies foi relatada anteriormente por Miller (1997), que revisou dados sobre nidificação de diversas populações. As diferenças para o tamanho da ninhada entre as espécies podem ser explicadas por características genéticas das fêmeas (Tiwari \& Bjorndal 2000).

Adicionalmente, outros estudos indicam diferenças para o tamanho das ninhadas entre populações (Tabela 2). Na Praia do Forte, localizada no litoral norte da Bahia, entre 1987 e 1993 (Marcovaldi \& Laurent 1996), foram verificados tamanhos de ninhadas maiores do que os registrados no presente estudo. Estas diferenças entre as populações podem ter ocorrido devido às diferenças no número amostral de ninhos e de temporadas reprodutivas considerados em ambas as regiões comparadas. Também poderiam ser explicadas pela quantidade de energia alocada para produção de ovos durante cada temporada (Tiwari \& Bjorndal 2000).

Os sucessos médios de eclosão registrados no sul da Bahia foram inferiores aos obtidos em outros locais. As ninhadas de ovos de tartarugas marinhas tipicamente têm sucesso de eclosão superior a $80 \%$ (Miller 1997), mas estudos realizados in situ dificilmente registram sucessos médios tão altos (Tabela 2). O sucesso de eclosão e a mortalidade embrionária variam de acordo com características ambientais durante o período de incubação, que in uenciam principalmente a temperatura e a umidade no interior do ninho (Ackerman 1997). Além disso, inundações pela maré e infestações por fungos e bactérias também podem in uenciar a taxa de eclosão dos ninhos (Wyneken et al. 1988, Phillot et al. 2001).

No presente estudo, tanto para os ninhos de $C$. caretta quanto para os ninhos de E. imbricata, a proporção de ovos classificados como não desenvolvidos foi maior do que as demais categorias de mortalidade embrionária. Os ovos não desenvolvidos podem se tratar de ovos inférteis ou com mortalidade embrionária precoce. De acordo com Wyneken et al. (1988), como os ninhos são abertos apenas após 50-70 dias de incubação, a mortalidade embrionária precoce pode ser mascarada pela decomposição e pode não ser distinguível da infertilidade verdadeira. Estudos em laboratório e em campo mais criteriosos, contudo, indicaram que a fertilidade dos ovos de tartarugas marinhas é superior a 90\% (Whitmore \& Dutton 1985, Wyneken et al. 1988), provavelmente, portanto, grande parte dos ovos classificados como não desenvolvidos continham embriões mortos em fases iniciais de desenvolvimento.

Os resultados do presente estudo a respeito da mortalidade embrionária podem estar relacionados ao tempo de duração de cada fase. Considerando-se os estágios de desenvolvimento embrionários propostos por Miller (1985) e utilizando os dados de maior e menor duração de incubação para o cálculo dos dias de cada fase de incubação (Miller refere-se a porcentagens da duração da incubação ao invés dos dias exatos), verificou-se que a fase de ovos não desenvolvidos, dura aproximadamente 9 dias no oviduto e entre 7 e 9 dias após a ovoposição, totalizando entre 16 e 18 dias; a fase 1, dura entre 10 e 14 dias, a fase 2 , entre 9 e 12 dias; e a fase 3 , entre 20 e 27 dias. 
O fato do estágio de desenvolvimento embrionário considerado como intermediário (fase 2) no presente estudo apresentar um período de duração relativamente menor em relação às demais fases, explicaria, portanto, a menor mortalidade embrionária verificada nesta fase para ambas as espécies. Resultado semelhante foi encontrado em outros locais para D. coriacea (Whitmore \& Dutton 1985, Bell et al. 2003) e para C. mydas (Whitmore \& Dutton 1985). Por outro lado, a fase de desenvolvimento embrionário tardio (fase 3), é aquela em que os embriões estão menos sujeitos à mortalidade embrionária, pois apresentou proporcionalmente taxas de mortalidade similares às das demais fases, apesar da sua maior duração em relação às demais.

Diferentes populações reprodutivas também apresentam diferenças nas durações da incubação, por exemplo, as durações médias da incubação registradas neste estudo foram maiores que aquelas registradas para os ninhos da Praia do Forte, no litoral norte da Bahia (Tabela 2). A duração da incubação está diretamente relacionada à temperatura de incubação, a qual por sua vez in uencia características dos filhotes, sendo a determinação sexual a mais importante delas (Davenport 1997). Portanto, a maior duração da incubação no litoral sul da Bahia em relação aos dados registrados para a os ninhos da Praia do Forte, pode levar a uma maior produção de filhotes machos. A duração de incubação que gera $50 \%$ de machos e $50 \%$ de fêmeas, denominada duração pivotal, foi estimada em 59,3 dias para C. caretta (Marcovaldi et al. 1997) e em 62,8 dias para E. imbricata (Godfrey et al. 1999), em estudos realizados com as populações do norte da Bahia. Embora a duração média da incubação na área de estudo tenha sido inferior à duração pivotal, ela é maior do que a duração no litoral norte da Bahia. Uma vez que, quanto maior a temperatura e, portanto, menor a duração da incubação, maior a produção de fêmeas (Yntema \& Mrosovsky 1982), os resultados ora apresentados sugerem que o litoral sul da Bahia gera mais machos em relação ao litoral norte deste estado. De fato já foi verificado que nas áreas de nidificação de $C$. caretta no litoral do Espírito Santo as durações médias da incubação são mais altas do que no litoral norte da Bahia, resultando em uma maior produção de filhotes machos (Baptistotte et al. 1999). O litoral norte da Bahia é a principal área de nidificação de C. caretta e E. imbricata no Brasil e cerca de $90 \%$ dos filhotes nascidos nestas áreas são fêmeas (Marcovaldi et al. 1997, Godfrey et al. 1999). Portanto, áreas localizadas mais ao sul e com durações médias da incubação maiores - e, portanto, temperaturas de incubação mais baixas -, como é o caso do litoral do Espírito Santo, para C. caretta, e do litoral sul da Bahia, para $C$. caretta e E. imbricata, são áreas relevantes para a conservação, visto que auxiliam na manutenção da saúde demográfica das populações dessas espécies que se reproduzem no Brasil.

Além disso, as populações de tartarugas marinhas são vulneráveis a diferentes ameaças, como destruição e degradação de habitat (Lagueux et al. 2003), desenvolvimento costeiro (Meylan \& Donnelly 1999, Kudo et al. 2003), captura incidental na pesca (Lewinson et al. 2004, Marcovaldi et al. 2006) e mudanças climáticas (Glen \& Mrosovsky 2004, Weishampel et al. 2004), de modo que a proteção de diferentes habitats de nidificação é essencial para a conservação dessas espécies, pois aumentam a variabilidade genética e fenotípica das populações, tornando-as mais aptas a enfrentar tais ameaças.

Portanto, constata-se que mesmo áreas com menor concentração de ninhos, como é o caso do litoral sul da Bahia, devem ser alvo de esforços para a conservação dessas espécies ameaçadas. Desta forma, medidas devem ser adotadas para proteção destes locais de nidificação. O litoral sul da Bahia vem passando nos últimos anos por um rápido processo de ocupação turística, especialmente após a construção da rodovia que liga Ilhéus a Itacaré (BA-001). As poucas ações para proteção destas espécies estão sendo realizadas de forma isolada por parte de algumas organizações não governamentais e instituições privadas, como o Projeto Txaitaruga em Itacaré e o Projeto Ecotuba em Una, uma região mais ao sul do estado da Bahia. Os dados sugerem a importância de uma ação oficial e coordenada, como o estabelecimento de parcerias sólidas com o Centro TAMAR/ ICMBio (Instituto Chico Mendes de Conservação da Biodiversidade). Além disso, recomenda-se que o Centro TAMAR/ICMBio, instituição governamental responsável pela conservação das tartarugas marinhas, exerça a fiscalização e acompanhamento constante das atividades desenvolvidas pelos seus parceiros, a fim de evitar que suas ações causem danos às populações de tartarugas marinhas, como alterações na determinação sexual.

\section{Agradecimentos}

Os autores agradecem a Alvimar Valadares (Capixaba) e Eric Macedo pelo auxílio no monitoramento das praias, ao Txai Resort pelo apoio logístico e financeiro, à Fundação de Amparo a Pesquisa do Estado da Bahia (FAPESB) pela concessão de bolsa de mestrado (CSC) e ao Projeto TAMAR/ICMBio pelo apoio técnico.

\section{Referências}

ACKERMAN, R.A. 1997. The nest environment and the embryonic development of sea turtles. In The biology of sea turtles (P.L. Lutz \& J.A. Musick, eds). CRC Press, Boca Raton, p. 83-106.

ALMEIDA, A.P. \& MENDES, S.L. 2007. An analysis of the role of local fishermen in the conservation of the loggerhead turtle (Caretta caretta) in Pontal do Ipiranga, Linhares, ES, Brazil. Biol. Conserv. 134(1):106-112.

BAPTISTOTTE, C., SCALFONE, J.T. \& MROSOVSKY, N. 1999. Maleproducing thermal ecology of a southern turtle nesting beach in Brazil: implications for conservation. Anim. Conserv. 2(1):9-13.

BAptistotte, C., THOMÉ, J.C.A. \& BJORNDAL, K.A. 2003. Reproductive biology and conservation status of the loggerhead sea turtle (Caretta caretta) in Espírito Santo Estate, Brazil. Chelonian Conserv. Biol. 4(3):523-529.

BELL, B.A., SPOTILA, J.R., PALADINO, F.V. \& REINA, R.D. 2003. Low reproductive success of leatherback turtle, Dermochelys coriacea, is due to high embryonic mortality. Biol. Conserv. 115(1):131-138.

BELLINI, C. 1996. Reproduction and feeding of marine turtles in the Fernando de Noronha Archipelago, Brazil. Mar. Turtle Newsl. 74:12-13.

BELlini, C., MARCOVALDI, M.A., SANCHES, T.M., GROSSMAN, A. \& SALES, G. 1996. Atol das Rocas Biological Reserve: second largest Chelonia rookery in Brazil. Mar. Turtle Newsl. 72:1-2.

Centro de Proteção à Tartarugas Marinhas - TAMAR; Instituto Brasileiro do Meio Ambiente e dos Recursos Renováveis - IBAMA; Ministério do Meio Ambiente - MMA. 2006. Relatório Técnico Anual Bahia-Junho de 2005 a Maio de 2006. Salvador: TAMAR.

DAVENPORT, J. 1997. Temperature and the life-history strategies of sea turtles. J. Therm. Biol. 22(6):479-488.

ECKERT, K.L. \& ECKERT, S.A. 1990. Embryo mortality and hatch success in situ and translocated leatherback sea turtle Dermochelys coriacea eggs. Biol. Conserv. 53(1):37-46.

FERREIRA-JUNIOR, P.D., CASTRO, P.T.A., ADDAD, J.E. \& LORENZO, M. 2003. Aspectos fisiográficos das áreas de nidificação da tartaruga marinha Caretta caretta na praia da Guanabara, Anchieta, Espírito Santo. Publ. Avulsas Inst. Pau-Brasil, 7:1-16.

GLEN, F. \& MROSOVSKY, N. 2004. Antigua revisited: the impact of climate change on sand and nest temperature at a hawksbill turtle (Eretmochelys imbricata) nesting beach. Global Change Biol. 10(12):2036-2045.

GODFREY, M.H., D’AMATO,A.F., MARCOVALDI, M.A. \& MROSOVSKY, N. 1999. Pivotal temperature and predicted sex ratios for hatchlings hawksbill turtles from Brazil. Can. J. Zool. 77(9):1465-1473. 
Instituto Brasileiro do Meio Ambiente e dos Recursos Renováveis - IBAMA 2003. Lista nacional das espécies da fauna brasileira ameaçadas de extinção. Disponível em: <http://www.ibama.gov.br>. Acesso em: 22/07/2007.

KUDO, H., MURAKAMI, A. \& WATANABE, S. 2003. Effects of sand hardness and human beach use on emergence success of loggerhead se turtles on Yakushima Island, Japan. Chelonian Conserv. Biol. 4(3):695-696.

LAGUEUX, C.J., CAMPBELL, C.L. \& McCOY, W.A. 2003. Nesting and conservation of the hawksbill turtle, Eretmochelys imbricata, in the Pearl Cays, Nicaragua. Chelonian Conserv. Biol. 4(3):588-602.

LEWINSON, R.L., FREEMAN, S.A. \& CROWDER, L.B. 2004. Quantifying the effects of fisheries on threatened species: the impact of pelagic longlines on loggerhead and leatherback sea turtles. Ecol. Lett. 7(3):221-231.

MARCOVALDI, M.A. \& LAURENT, A. 1996. A six season study of marine turtle nesting at Praia do Forte, Bahia, Brazil, with implications for conservation and management. Chelonian Conserv. Biol. 2(1):55-59.

MARCOVALDI, M.A. \& MARCOVALDI, G.G. 1999. Marine turtles of Brazil: the history and structure of Projeto TAMAR-IBAMA. Biol. Conserv. 91(1):35-41.

MARCOVALDI, M.A., GODFREY, M.H. \& MROSOVSKY, N. 1997. Estimating sex ratios of loggerheads turtles in Brazil from pivotal incubation durations. Can. J. Zool. 75(5):755-770.

MARCOVALDI, M.A., VIEITAS, C.F. \& GODFREY, M.H. 1999. Nesting and conservation management of hawksbill turtles (Eretmochelys imbricata) in Northern Bahia, Brazil. Chelonian Conserv. Biol. 3(2):301-307.

MARCOVALDI, M.A., SALES, G.S., THOMÉ, J.C.A., DIAS Da SILVA, A.C.C., GALLO, B.M.G., LIMA, E.H.S.M., LIMA, E.P. \& BELLINI, C. 2006. Sea turtle and fishery interactions in Brazil: identifying and mitigating potential con icts. Mar. Turtle Newsl. 112:4-8.

MARCOVALDI, M.A., LOPEZ, G.G., SOARES, L.S., SANTOS, A.J.B., BELLINI, C. \& BARATA, P.C.R. 2007. Fifteen years of hawksbill sea turtle (Eretmochelys imbricata) nesting in Northern Brazil. Chelonian Conserv. Biol. 6(2):223-228.

MASCARENHAS, R., ZEPPELINI FILHO, D. \& MOREIRA, V.S. 2003. Observations on sea turtles in the state of Paraíba, Brazil. Mar. Turtle Newsl. 101:16-18.
MEYLAN, A.B. \& DONNELLY, M. 1999. Status justification for listing the hawksbill turtle (Eretmochelys imbricata) as critically endangered on the 1996 IUCN Red List of Threatened Animals. Chelonian Conserv. Biol. 3(2):200-224.

MILLER, J.D. 1985. Embriology of marine turtles. In Biology of Reptilia (C. Gans \& F. Billet, eds). John Wiley and Sons, New York, p. 270-328.

MILLER, J.D. 1997. Reproduction in sea turtles. In The biology of sea turtles (P.L. Lutz \& J.A. Musick, eds). CRC Press, Boca Raton, p. 51-81.

PHILLOTT, A.D., PARMENTER, C.J. \& LIMPUS, C.J. 2001. Myco ora identified from failed green (Chelonia mydas) and loggerhead (Caretta caretta) sea turtle eggs at Heron Island, Australia. Chelonian Conserv. Biol. 4(1):170-172.

TIWARI, M. \& BJORNDAL, K.A. 2000. Variation in morphology and reproduction in loggerheads, Caretta caretta, nesting in the United States, Brazil and Greece. Herpetologica, 56(3):343-356.

THOMÉ, J.C.A., BAPTISTOTTE, C., MOREIRA, L.M.P., SCALFONI, J.T., ALMEIDA, A.P., RIETH, D.B. \& BARATA, P.C.R. 2007. Nesting biology and conservation of the leatherback sea turtle (Dermochelys coriacea) in the State of Espirito Santo, Brazil, 1988-1989 to 2003-2004. Chelonian Conserv. Biol. 6(1):15-27.

WEISHAMPEL, J.F., BAGLEY, D.A. \& EHRHART, L.M. 2004. Earlier nesting by loggerhead sea turtles following sea surface warming. Glob. chang. biol. 10(8):1424-1427.

WHITMORE, C.P. \& DUTTON, P.H. 1985. Infertility, embryonic mortality and nest-site selection in leatherback and green sea turtles in Suriname. Biol. Conserv. 34(3):251-272.

WYNEKEN, J., BURKE, T.J., SALMON, M. \& PEDERSEN, D.K. 1988. Egg failure in natural and relocated sea turtle nests. J. Herpetol. 22(1):88-96.

YNTEMA, C.L. \& MROSOVSKY, N. 1982. Critical periods and pivotal temperatures for sexual differentiation in loggerhead sea turtles. Can. J. Zool. 60(5):1012-1016.

Recebido em 24/10/08 Versão Reformulada recebida em 29/04/09 Publicado em 15/05/09 